\title{
Lung ultrasound artifacts in COVID-19 patients
}

\author{
Christine McElyea ${ }^{1}$ (D) Christopher Do ${ }^{1} \cdot$ Keith Killu $^{1}$
}

Received: 18 May 2020 / Accepted: 12 August 2020 / Published online: 25 August 2020

(c) Società Italiana di Ultrasonologia in Medicina e Biologia (SIUMB) 2020

\begin{abstract}
Lung ultrasound is an essential tool in critical care, made more so by the enhanced precautions associated with the Covid-19 pandemic. Here we describe 2 cases of multiple, small shred signs seen on ultrasound of Covid-19 patients.
\end{abstract}

Keywords COVID-19 $\cdot$ Lung ultrasound $\cdot$ Shred sign

\section{Introduction}

Lung ultrasound is an essential tool in identifying lung disease and most practitioners will be able to identify pathology not visible on chest X-Ray [1]. As of the summer of 2020, there are more than 12.5 million reported cases of COVID19 caused by the Coronavirus 2 (SARS-CoV-2) causing a pandemic that has presented many challenges in the traditional approach to patients with hypoxemia and shortness of breath or respiratory failure. The disease has affected over 188 countries and reported deaths are over 500,000 so far across the globe [2,3]. Traditional radiologic imaging for patients who present with suspected pneumonia, including chest X-ray or CT chest is not routinely recommended to limit healthcare worker exposure. Using bedside ultrasound, with the appropriate precautions, can help the practitioner significantly in identifying the lung pathology [4]. Studies describing lung ultrasound findings have been increasing over the last 25 years, with more recent articles describing the use of ultrasound in a standardized fashion to identify lung disease in COVID-19 era [5].

Christine McElyea

Christine.mcelyea@med.usc.edu

1 Department of Pulmonary, Critical Care and Sleep Medicine, University of Southern California, 2020 Zonal Avenue, IRD 723, Los Angeles, CA 90033, USA

\section{Case reports}

\section{Patient A}

We present the case of a 64-year-old woman with a past medical history of developmental delay with associated dementia and schizoaffective disorder, and recurrent urinary tract infections who was sent to the emergency department from her skilled nursing facility for fevers of $38.7{ }^{\circ} \mathrm{C}$. Initial complete blood count demonstrated leukocytosis to 14.5 with $8 \%$ bands. A CT of the abdomen and pelvis demonstrated bladder wall thickening concerning for cystitis; she was admitted and treated with ceftriaxone for presumed urinary tract infection. By day 4 of admission, her fevers had failed to remit, and a CT of the chest was obtained which demonstrated bilateral, patchy ground-glass opacities. A SARS CoV-2 PCR was sent and returned positive on day 7 of admission at which time she was started on azithromycin and hydroxychloroquine. She developed worsening hypoxic respiratory failure and was intubated on day 13 of admission and transferred to our facility for further care. Of note, her nursing home roommate was also admitted to our ICU with the novel coronavirus. Lung ultrasound was used in addition to the earlier conventional modalities of chest X-Ray and CT scan. She received one dose of tocilizumab without significant improvement in her respiratory status and after further decline was transitioned to comfort-focused measures by her family and passed away.

\section{Patient B}

A 76-year-old female with a past medical history of Type 2 Diabetes who presented with 2 weeks of body aches, fevers, 
cough, and progressive shortness of breath; with several family members experiencing similar symptoms at home. In the emergency department, she was noted to be tachypneic with pulse oximetry as low as the mid-50 s. A chest x-ray demonstrated bibasilar infiltrates. She was initially placed on a non-rebreather mask at $15 \mathrm{~L}$ per minute, then high flow nasal cannula with oxygen saturation improving to $90 \%$. A SARS CoV 2 PCR was sent, subsequently returning positive, and she was admitted to the progressive care unit where her respiratory status remained tenuous. Several goals of care meetings were held with the patient and her care teamshe initially endorsed do not resuscitate and do not intubate status, given this, in combination with worsening hypoxia, she was given one dose of $400 \mathrm{mg}$ IV tocilizumab on day 2 of admission. By day 4 of admission, her high flow nasal cannula had increased to $60 \mathrm{~L}$ per minute of flow with $\mathrm{FiO} 2$ $95 \%$ and oxygen saturation in the $80 \mathrm{~s}$. At this time, she requested alteration of her code status to full care-she then transferred to the intensive care unit, underwent endotracheal intubation, and was placed on mechanical ventilation. Ultrasound exam of her lungs was performed on the day of intubation.

Lung ultrasound for both patients was performed using a FujiFilm Sonosite X-Port machine (Seattle) with a linear transducer with a frequency of $13 \mathrm{MHz}$. The B Mode was used with a depth dependent on the patient's chest wall size, ranging between 3.6 and $4.4 \mathrm{~cm}$, with optimal gain at 0.5 and the mechanical index was set low at 0.5. Full contact and droplet precautions and barriers were applied.

Both patients were in a supine position with the head of the bed at $30^{\circ}$ angle. Anterior lung fields were examined to cover most lung zones [6]. Eight (8) zones were examined in total. Four (4) for each lung. Left upper and lower anterior and left upper and lower lateral chest, right upper and lower anterior and right upper and lower lateral chest Fig. 1.

Findings of the ultrasound exam on both patients were the following: see Fig. 2

1. Skip areas of normal pleural lines throughout both lung fields.

2. Thickened pleural lines in areas with multiple subpleural small areas of hypoechoic shredded lung parenchyma of different measurements ranging between 0.25 and $0.5 \mathrm{~cm}$ in depth or sometimes larger, representing multiple shred signs or C-profile Fig. 2b, b'.

3. The pleural line is of the grainy appearance of $1-2 \mathrm{~mm}$ grains in diameter. The grains are of hyperechoic appearance. This is different from and more than the classical C-profile that represents consolidations. The image resembles grouped stone pebbles Fig. 2c, c'.

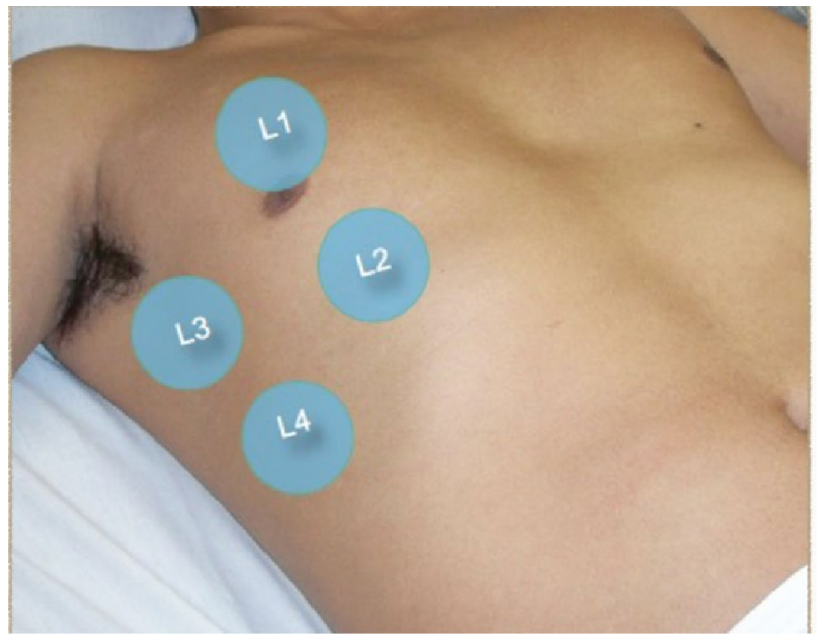

Fig. 1 Lung zones in a supine position. L1 Upper anterior lung zone. L2 Lower anterior lung zone. L3 Upper lateral lung zone. L4 Lower lateral lung zone

4. B-Lines noted scattered throughout both lungs in a heterogeneous pattern Fig. 2d.

5. Minimal pleural effusions bilaterally.

6. Lateral lower zones were showing atelectasis and minimal pleural effusions.

In patient $\mathrm{A}$, the findings described were noted in the upper anterior lung zones bilaterally, while a chest $\mathrm{x}$-ray obtained concurrently demonstrated infiltrate in bilateral lower lateral lung zones. In the right and left lateral lower zones, there was atelectasis noted by ultrasound. In patient $\mathrm{B}$, there was a consolidation of both lower lung zones corresponding to the areas examined by ultrasound showing shred signs, with the pebbles appearance scattered on the upper anterior lung zones Fig. 2.

Some of the findings listed above were described by recent articles using ultrasound in COVID-19 patients [7]. In this article, we are listing the findings with a more detailed description and emphasis on possible early changes and their characters that could be unique for this disease.

\section{Discussion}

COVID-19 patients can present in different stages with different signs, symptoms, and radiological criteria [8]. It is estimated that over $17 \%$ of infected individuals, who present with mild to moderate disease may not show any radiological signs on chest X-ray [9]. Application of bedside lung POCUS is well established, and many studies have shown 
A

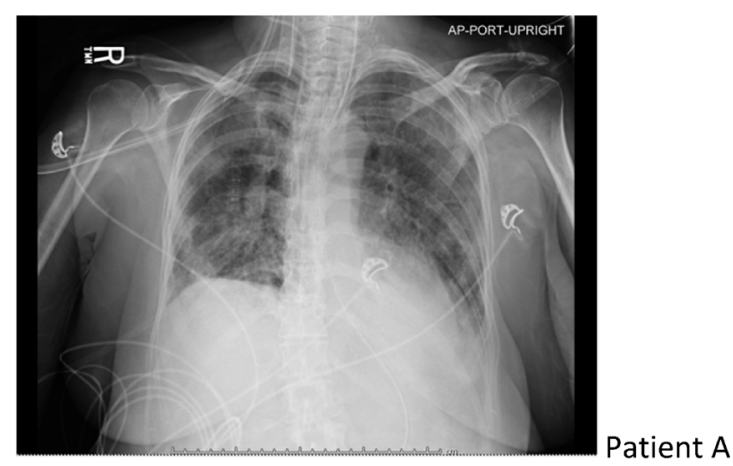

B

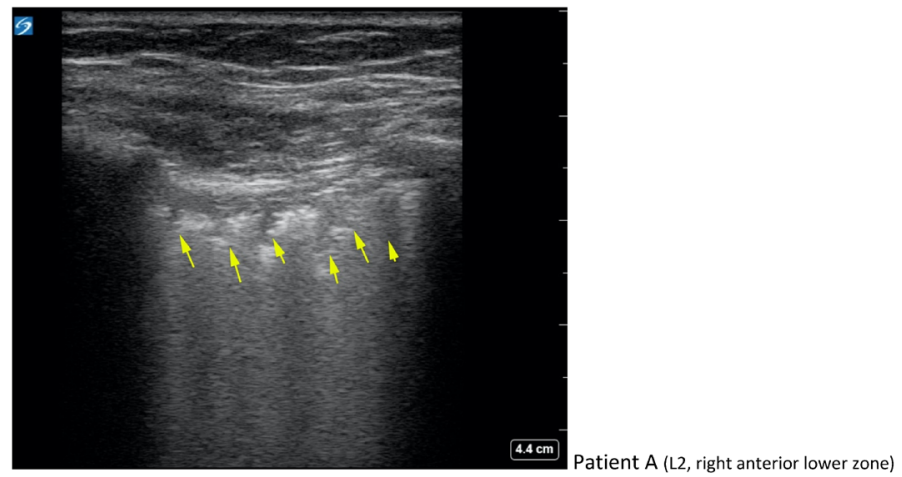

$A^{\prime}$

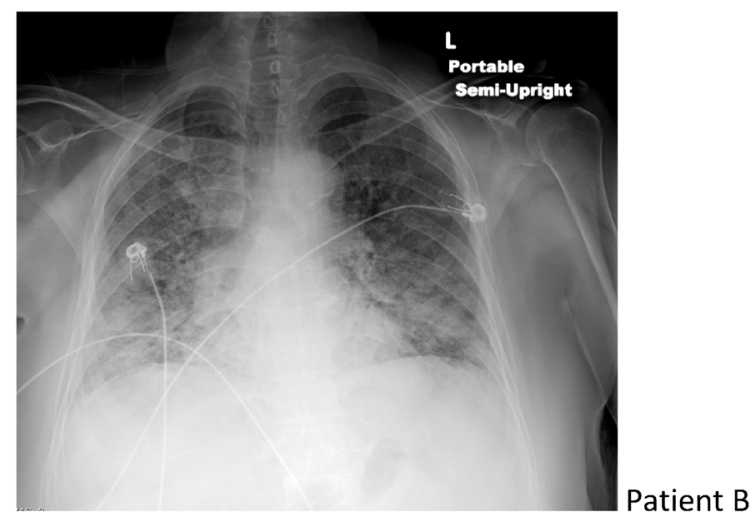

$B^{\prime}$

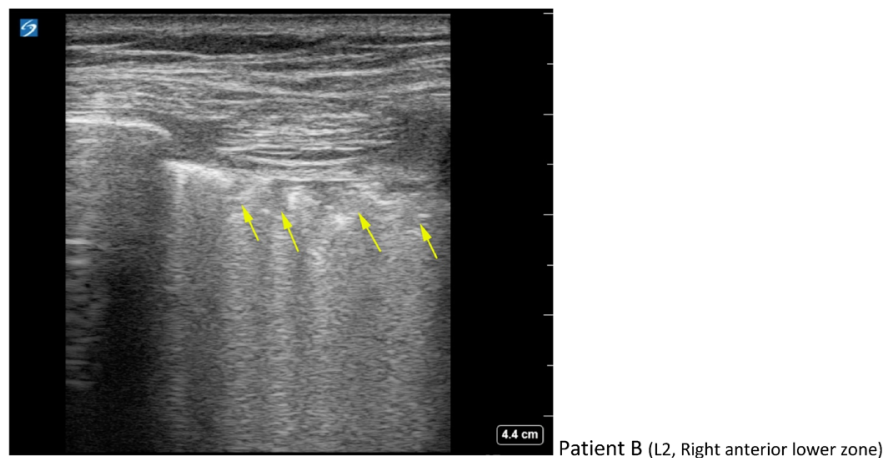

Fig. 2 a, a' Patient A and B chest X-ray prior to lung ultrasound with bibasilar infiltrates. b, b' Pleural lines for patients A and B with multiple small shred signs (arrows). c, c' illustrating the grainy appear-

effectiveness in diagnosis and management [1]. Detecting lung consolidation and interstitial lung disease can be achieved effectively using lung US [10]. Using bedside ultrasound to help manage patients with COVID-19 is becoming more prevalent and effective. We presented two cases of COVID-19 with acute respiratory failure, one with nearnormal chest X-ray and the other with bilateral infiltrates. Using bedside lung POCUS helped us identify the severity of the disease, as well as confirm the clinical diagnosis of a pneumonic process. Unable to perform daily CXR, and or CAT scans of the chest due to the infectious process and limitation of isolation and avoiding excessive exposure, lung POCUS can be performed initially and early in the disease process when it can detect abnormalities not detected by CXR, and can assist in daily management as well.

We noted some findings that could be specific for the disease or representing a continuum of alveolar interstitial lung disease. The thickening of the pleural line was noted with skip areas of normal pleura. Shred signs tended to ance of the pleural line resembling pebbles in patients A and B, identified by the arrows. d Showing skip areas of normal to mildly thickened pleural line with multiple B-Lines (arrow heads)

develop in multiple fashion as small aggregates, affecting mainly anterior upper zones, and they were not seen as the usual bacterial lobar pneumonia, involving larger areas of consolidations. Areas resembling Pebbles were noted on the ultrasound and described above, need to be investigated further, its significance could represent the loss of integrity to lung tissue secondary to poor blood flow distribution as hypothesized by some researchers [11], and our hypothesis also supported by previous older and some recent literature $[12,13]$, most probably representing the first manifestation of the known Shred sign as an evolving pneumonic process at different stages since it is close to areas where the shred signs were developing, Fig. 3. We were able to capture these early changes since ultrasound was used earlier in COVID patients as we suspect pneumonia in those patients even with a normal CXR as inpatient $\mathrm{A}$, representing part of the early interstitial and parenchymal lung disease and the early manifestation and development of shred sign, not described in previous literature. This may help investigators 


\section{C}

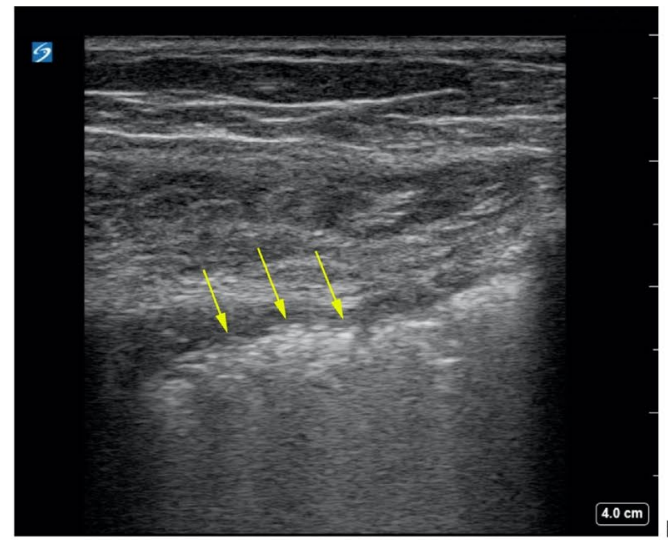

$4.0 \mathrm{~cm}$

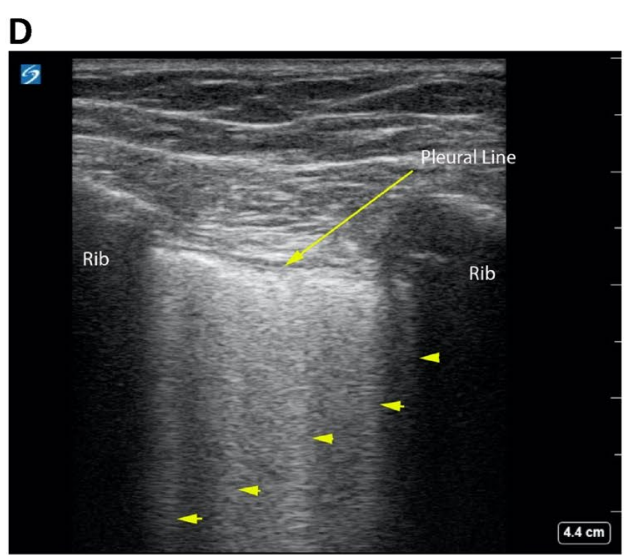

$\mathbf{C}^{\prime}$

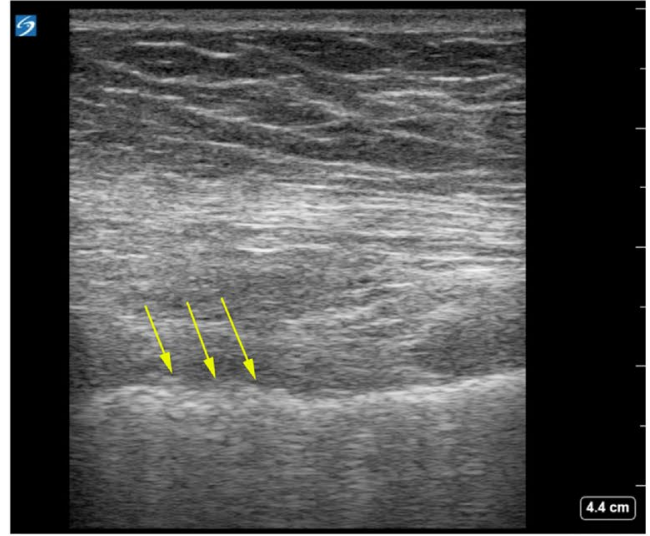

Patient B (L1, left anterior upper zone)

Fig. 2 (continued)

and clinicians identify patients with early pneumonia without other radiological evidence.

Using higher frequency transducer helped us identify these findings, which sometimes aren't visible, using the traditional phased array transducer with the usual $12-15 \mathrm{~cm}$ depth.

The advantages of ultrasound in this pandemic are the ease of use, the reproducibility of the testing, and limiting radiation exposure, infection, and transmission of disease. Other advantages are clear in limited-resource areas as well as in pregnant women when limiting radiation exposure $[14,15]$.
Limitations to our exams were not including the posterior lower lobes in the back. This was not done to limit the study time and decrease exposure. Other inherent limitations include the possibility of cross-contamination of other individuals if cleaning and isolation precautions are not followed properly, and operator variability. We recommend using a dedicated ultrasound machine to perform these exams.

Further research and studies need to be done to verify these findings and investigate the changes noted in our ultrasound exam. 


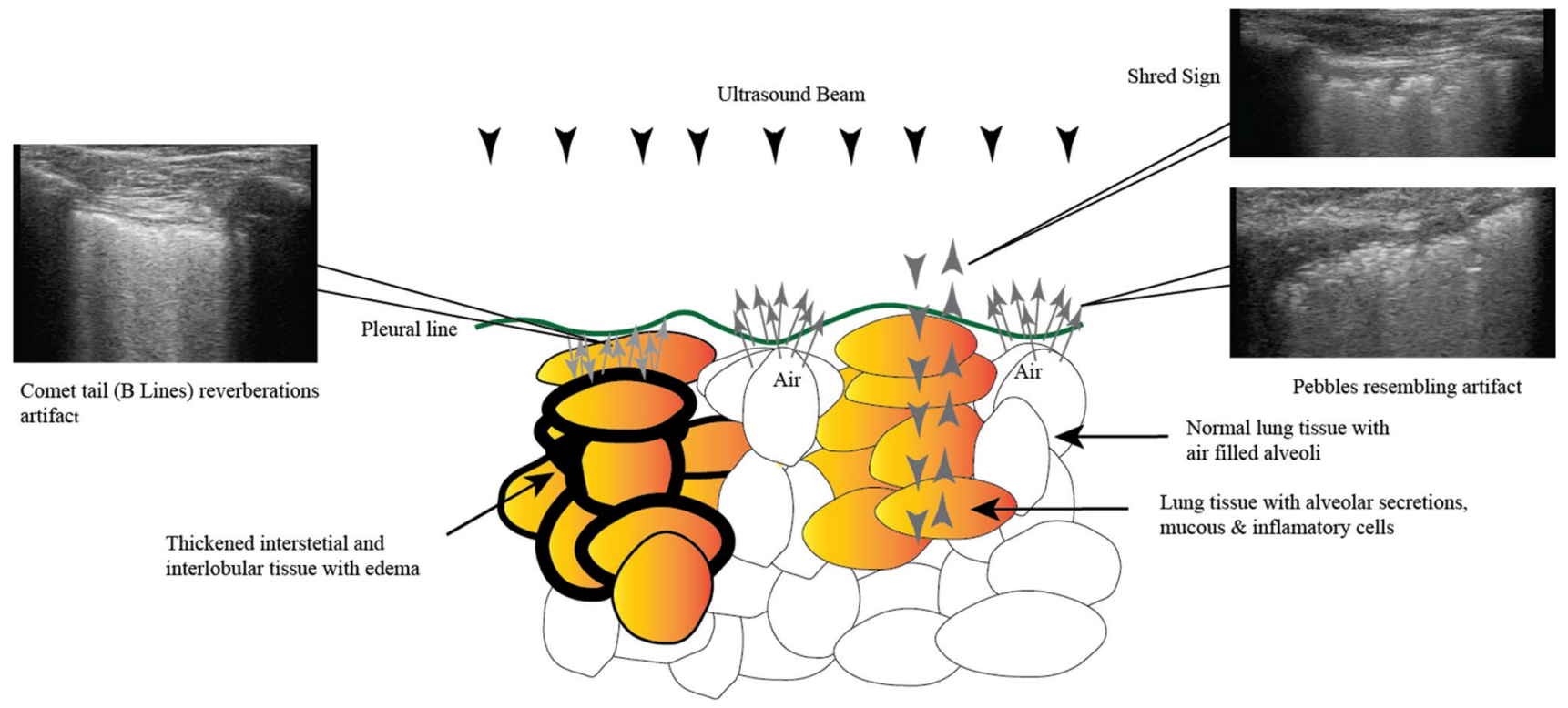

Fig. 3 Illustration of the pathophysiology of the different ultrasound signs including the pebbles resembling artifacts. Note areas of normal lung parenchyma and air-filled alveoli with poor transmission to ultrasound, reflecting and scattering the waves (Arrows) as bright pebbles like artifacts. Also, areas of lung tissue with early buildup and accumulation of alveolar secretions, mucous and inflammatory cells, leading to transmission of the ultrasound beam and its refraction to deeper tissues (arrow heads). Thickening of the interstitial layer with fluid accumulation can lead to the reverberation of the ultrasound beam between the pleural line and the interlobular septa, leading to the development of the comet tail, or B Lines on the ultrasound screen
Funding The authors of this study declare no financial support was received for this study.

\section{Compliance with ethical standards}

Ethical standards This manuscript was written following all the ethical standards and full compliance required by this journal.

\section{References}

1. Tierney DM et al. (2020) Comparative performance of pulmonary ultrasound, chest radiograph, and CT among patients with acute respiratory failure. Crit Care Med 48(2):151-157. ISSN 1530-0293. Disponível em https://www.ncbi.nlm.nih.gov/pubme $\mathrm{d} / 31939782$.

2. https://www.covid19treatmentguidelines.nih.gov/overview/. NIH Coronavirus disease 2019 (COVID-19) treatment guidelines. NIH. gov, 2020.

3. Center JHU, A. M. C. R. Johns Hopkins University and Medicine/ Coronavirus Resource Center: https://coronavirus.jhu.edu 2020.

4. Buonsenso D et al. Point-of-care lung ultrasound findings in novel coronavirus disease-19 pnemoniae: a case report and potential applications during COVID-19 outbreak. Eur Rev Med Pharmacol Sci 24(5):2776-2780, 03 2020. ISSN 2284-0729. Disponível em: https://www.ncbi.nlm.nih.gov/pubmed/32196627.

5. Soldati $\mathrm{G}$ et al. Proposal for international standardization of the use of lung ultrasound for patients with COVID-19: a simple, quantitative, reproducible method. J Ultrasound Med
39(7):1413-1419, Jul 2020. ISSN 1550-9613. Disponível em: https://www.ncbi.nlm.nih.gov/pubmed/32227492.

6. Killu K the ICU Ultrasound Pocket Book. United States, p. 272 pages, 2015. Disponível em: https://books.apple.com/us/book/theicu-ultrasound-pocket-book/id957153627.

7. Soldati G et al. (2020) Is there a role for lung ultrasound during the COVID-19 pandemic? J Ultrasound Med 39(7):1459-1462. ISSN 1550-9613. Disponível em: https://www.ncbi.nlm.nih.gov/ pubmed/32198775.

8. Zhu N et al. (2019) A novel coronavirus from patients with pneumonia in China, 2019. N Engl J Med 382(8):727-733, 022020. ISSN 1533-4406. Disponível em: https://www.ncbi.nlm.nih.gov/ pubmed/31978945.

9. Guan WJ, Zhong NS (2020) Clinical characteristics of covid-19 in China. Reply. N Engl J Med 382. ISSN 1533-4406. Disponível em: https://www.ncbi.nlm.nih.gov/pubmed/32220206.

10. Lichtenstein DA, Mezière GA (2008) Relevance of lung ultrasound in the diagnosis of acute respiratory failure: the BLUE protocol. Chest 134(1): 117-25. ISSN 0012-3692. Disponível em: https://www.ncbi.nlm.nih.gov/pubmed/18403664.

11. Gattinoni L et al. (2020) Covid-19 does not lead to a "Typical" acute respiratory distress syndrome. Am J Respir Crit Care Med. ISSN 1535-4970. Disponível em: https://www.ncbi.nlm.nih.gov/ pubmed/32228035.

12. Jambrik, Z. et al. (2004) Usefulness of ultrasound lung comets as a nonradiologic sign of extravascular lung water. Am J Cardiol 93(10): 1265-70. ISSN 0002-9149. Disponível em: https://www. ncbi.nlm.nih.gov/pubmed/15135701.

13. Soldati $\mathrm{G}$ et al. (2019) The role of ultrasound lung artifacts in the diagnosis of respiratory diseases. Expert Rev Respir Med 13(2): 163-172, 02 2019. ISSN 1747-6356. Disponível em: https://www. ncbi.nlm.nih.gov/pubmed/30616416. 
14. Buonsenso D (2020) et al. Effectiveness of rapid lung ultrasound training program for gynecologists and obstetricians managing pregnant women with suspected COVID-19. Ultrasound Obstet Gynecol 56(1):110-111, 07 2020. ISSN 1469-0705. Disponível em: https://www.ncbi.nlm.nih.gov/pubmed/32349175.

15. Buonsenso D et al. (2020) Social consequences of COVID-19 in a low resource setting in Sierra Leone, West Africa. Int J Infect Dis
97:23-26. ISSN 1878-3511. Disponível em: https://www.ncbi. nlm.nih.gov/pubmed/32497794.

Publisher's Note Springer Nature remains neutral with regard to jurisdictional claims in published maps and institutional affiliations. 